\title{
Joint optimization of source, mask, and pupil in optical lithography
}

\author{
Jia Li and Edmund Y. Lam \\ Imaging Systems Laboratory, Department of Electrical and Electronic Engineering, \\ The University of Hong Kong, Pokfulam, Hong Kong, China
}

\begin{abstract}
Mask topography effects need to be taken into consideration for more advanced resolution enhancement techniques in optical lithography. However, rigorous 3D mask model achieves high accuracy at a large computational cost. This work develops a combined source, mask and pupil optimization (SMPO) approach by taking advantage of the fact that pupil phase manipulation is capable of partially compensating for mask topography effects. We first design the pupil wavefront function by incorporating primary and secondary spherical aberration through the coefficients of the Zernike polynomials, and achieve optimal source-mask pair under the condition of aberrated pupil. Evaluations against conventional source mask optimization (SMO) without incorporating pupil aberrations show that SMPO provides improved performance in terms of pattern fidelity and process window sizes.
\end{abstract}

Keywords: Mask topography effects, spherical aberration, source mask optimization, usable depth of focus, computational lithography

\section{INTRODUCTION}

In ultra low-k1 era of optical lithography, one primary limitation of process window is the noticeable difference in best focus (BF) among various feature sizes. ${ }^{1}$ Rigorous 3D mask experiments have confirmed that mask topography is a leading cause due to the fact that the thickness of the mask absorber produces phase errors among different diffraction orders. ${ }^{2,3}$

Unfortunately, the rigorous electromagnetic field (EMF) modeling used to describe light diffraction from the mask generally involves intensive computation, ${ }^{4}$ which limits the wide adoption of rigorous 3D mask modeling for practical large layout simulations in advanced resolution enhancement techniques (RETs) such as source and mask optimization (SMO). ${ }^{5,6}$ Additionally, although SMO is a powerful and effective technique which provides more flexibility regarding both the mask design and illumination configuration adjustment, ${ }^{7-10}$ it is inadequate to control the phase in the lens pupil. In order to compensate for the phase errors induced by mask topography effects, additional degrees of freedom are required in terms of phase manipulation, namely to incorporate the phase parameter into source and mask optimization. ${ }^{11}$

In this work, a combined source, mask and pupil optimization (SMPO) approach is developed as an alternative to maximize the process window. Here, "alternative" has twofold meanings. First, unlike SMO, the proposed scheme takes advantage of the fact that pupil phase manipulation can partially compensate for thick mask topography effects. ${ }^{12}$ It incorporates some helpful pupil aberration terms into imaging systems of the optimization process, which makes the resulting source and mask robust against specific pupil aberrations, thereby being robust against similar imaging impact caused by mask topography. Second, the whole optimization procedure is performed based on the thin mask model, which makes sure that the speed is faster than that based on rigorous model. But to verify the practicability and effectiveness of the algorithm, we compare all the printed image fidelity and usable depth of focus (uDOF) through rigorous EMF simulations.

Section 2 contains a description of how to incorporate the pupil aberrations into SMPO strategy to compensate typical mask-induced effects. The application of the proposed approach to the test patterns and simulation results analysis are presented in Sec. 3 to demonstrate the enhancement on the image accuracy and uDOF.

Further author information: Edmund Y. Lam: E-mail: elam@eee.hku.hk

Optical Microlithography XXVII, edited by Kafai Lai, Andreas Erdmann, Proc. of SPIE Vol.

9052, 90520 S (C) 2014 SPIE · CCC code: 0277-786X/14/\$18 · doi: 10.1117/12.2045739

Proc. of SPIE Vol. 905290520 S-1 


\section{MUTUAL SOURCE MASK AND PUPIL OPTIMIZATION}

The objective of our work is to devise a method that incorporates pupil aberrations into source mask corrections to compensate for photomask topography degraded uDOF. In this section, we first model the imaging process with pupil aberrations, and then describe how to design a pupil aberration function including specific aberration terms to effectively compensate 3D mask effects. Next, under the circumstance of the aberrated transfer function, inverse optimization method is used to update the mask and source alternately until the termination criterion is reached..$^{13-16}$

\subsection{Forward Imaging Model with Aberrations}

The imaging process in computational lithography is frequently divided into two parts, namely the aerial image formation and photo-resist development. To include the pupil aberrations such that they occur in the practical lithography process, the aerial image $I_{a}(x, y)$ under a partially coherent illumination (PCI) system can be described as ${ }^{17}$

$$
I_{a}(x, y)=\int_{-\infty}^{\infty} \int_{\infty} J(f, g)\left|\int_{-\infty}^{\infty} \hat{H}\left(f+f_{1}, g+g_{1}\right) \mathrm{e}^{i 2 \pi W(\rho, \theta)} \hat{M}\left(f_{1}, g_{1}\right) \mathrm{e}^{-i 2 \pi\left(f_{1} x+g_{1} y\right)} \mathrm{d} f_{1} \mathrm{~d} g_{1}\right|^{2} \mathrm{~d} f \mathrm{~d} g .
$$

In Eq. (1), $\hat{M}$ represents the spectrum of input mask pattern $M(x, y)$, and $\hat{H}$ is the ideal pupil function of the optical system under nominal conditions. The wavefront aberration function, denoted by $W(\rho, \theta)$, is incorporated by multiplying an exponential term with it as power in the frequency domain. ${ }^{18,19}$ The function $J(f, g) \geq 0$ represents the effective source, which is normalized by its total energy. ${ }^{20,21}$

The aerial image $I_{a}(x, y)$ then goes through the photoresist development to form the printed image $I(x, y)$. Approximating the resist effect with a sigmoid function due to its differentiability ${ }^{22}$ the output $I(x, y)$ is given by

$$
I(x, y)=\operatorname{sig}\left\{I_{a}(x, y)\right\}=\frac{1}{1+\mathrm{e}^{-\alpha\left[I_{a}(x, y)-t_{r}\right]}},
$$

in which $t_{r}$ is the threshold and $\alpha$ indicates the steepness of the sigmoid function.

\subsection{Aberration-aware Pupil Function}

Deviation of wavefronts, also known as aberration, is recently developed in the extended Nijboer-Zernike theory, ${ }^{23}$ and circular wavefront profiles associated with aberrations can be mathematically modeled using Zernike polynomials, ${ }^{24}$ defined as

$$
W(\rho, \theta)=\sum_{i=-\infty}^{\infty} z_{i} F_{i}(\rho, \theta)
$$

where $F_{i}(\rho, \theta)$ is the $i$ th polynominal term with $z_{i}$ being the $i$ th Zernike coefficient. The Zernike coefficients are linearly independent, thus individual aberration contributions to an overall wavefront may be isolated and quantified separately. $\rho$ indicates the relative radial pupil position while $\theta$ is the polar angle, represented by

$$
\rho=\frac{\lambda}{\mathrm{NA}} \sqrt{f^{2}+g^{2}} \quad \text { and } \quad \theta=\arctan (g / f),
$$

where $\lambda$ is the incident light wavelength and NA is the numerical aperture.

Previous studies have found that mask topography have similar impact on the lithography imaging performance to that caused by wave aberrations. ${ }^{25,26}$ Consequently, the mask-induced phase deformation can be partially compensated by a manipulation of the pupil lens. ${ }^{12}$ Indeed, the simulation results exhibit that primary spherical aberrations (Zernike term $z_{9}$ ) have the highest sensitivity, which means a large impact on linewidth through focus, and 1D lines are also prone to be affected by other spherical aberrations such as secondary spherical (Zernike term $z_{16}$ ) since the spherical aberrations have radially dependent and rotationally symmetric 
form. ${ }^{26}$ The wavefront function $W(\rho, \theta)$ in this paper is therefore composed of primary and secondary spherical aberrations to further improve the imaging performance, as follows:

$$
W(\rho, \theta)=z_{9}\left(6 \rho^{4}-6 \rho^{2}+1\right)+z_{16}\left(20 \rho^{6}-30 \rho^{4}+12 \rho^{2}-1\right) .
$$

Here, we use $H\left(x, y ; z_{9}, z_{16}\right)$ to represent the aberrated point spread function with primary and secondary spherical aberrations, which can be obtained by taking the inverse Fourier transform $\left(\mathcal{F}^{-1}\right)$ of the pupil function with aberrations, i.e.,

$$
H\left(x, y ; z_{9}, z_{16}\right)=\mathcal{F}^{-1}\left\{\hat{H} \mathrm{e}^{i 2 \pi\left[z_{9}\left(6 \rho^{4}-6 \rho^{2}+1\right)+z_{16}\left(20 \rho^{6}-30 \rho^{4}+12 \rho^{2}-1\right)\right]}\right\} .
$$

After designing the pupil aberration function, we need to choose the Zernike coefficient values for the corresponding Zernike polynomials, which are the wavefront errors in wavelengths and are presented in units of waves. For instance, a coefficient of 0.1 means an aberration with the maximum value at $0.1 \lambda=0.1 \times 193 \mathrm{~nm}=19.3 \mathrm{~nm} .{ }^{26}$ The method of determining the Zernike coefficient values in our work is carrying out the exhaustive search on a series of target patterns, as described in Ref. 26, the exhaustive search is performed at different pitches to find an optimal coefficient $z_{9}$ which ensures the spherical aberration could be beneficial all of them. Thus we obtain an optimized value of $z_{9}$ and $z_{16}$ which just can counteract the opposite primary and secondary spherical aberrations induced by mask topography.

\subsection{Source Mask Optimization Framework for Fixed Aberrations}

In our approach, we make use of the relationship between the applied pupil filter and the aberration induced by the mask, which was claimed in Ref. 11, that is if an optimized pupil filter maximizes defocus-exposure window symmetry, then it would represent the inverse of the aberrations induced by mask.

Given two mask patterns, we not only consider the impact of the coefficient of primary spherical aberration on the compensation for 3D mask effects, but also select secondary spherical aberration to enable further tuning of the contributions of higher diffraction order. Assuming the optimal $z_{9}$ and $z_{16}$ have been found by an exhaustive grid search over the $z_{9}$ and $z_{16}$ parameter space, take $z_{9}=0.1$ and $z_{16}=-0.02$ as an example, which is consistent for mask topography inducing a $z_{9}=-0.1$ of primary spherical aberration and a $z_{16}=0.02$ of secondary spherical aberration. During the following process of source and mask optimization, the printed image $I\left(x, y ; z_{5}, z_{16}\right)$ is calculated under the circumstance of the fixed spherical aberrated pupil, rather than an ideal pupil without any aberrations. Hence, the optimum source-mask pair resulted from source mask optimization can effectively counteract the corresponding spherical aberration offset of $z_{9}=-0.1$ and $z_{16}=0.02$ induced by mask topography.

We generate the optimal source mask pair by minimizing the sum of the mismatches between the printed image and the desired one over all locations, to achieve the smallest accumulated pattern error. ${ }^{27}$ Mathematically, the SMPO minimization problem is formulated as

$$
\begin{array}{cl}
\underset{\mathbf{m}}{\operatorname{minimize}} & \left\|I\left(x, y ; z_{5}, z_{16}\right)-I_{0}(x, y)\right\|_{2}^{2} \\
\text { subject to } & M(x, y) \in\{0,1\}, \\
& J(f, g) \geq 0,
\end{array}
$$

Substituting the optimal $z_{9}$ and $z_{16}$ values into the above equation, then the current cost function can be expressed as

$$
\left\|\operatorname{sig}\left\{\sum_{f, g} J(f, g)\left|M(x, y) * \mathcal{F}^{-1}\left\{\hat{H} \mathrm{e}^{i 2 \pi\left[-0.1\left(6 \rho^{4}-6 \rho^{2}+1\right)+0.02\left(20 \rho^{6}-30 \rho^{4}+12 \rho^{2}-1\right)\right]}\right\}\right|^{2}\right\}-I_{0}(x, y)\right\|_{2}^{2} .
$$

The succeeding optimization is accomplished by conjugate-gradient (CG) method. ${ }^{28}$ The target pattern is assigned as the initial mask, and the first mask optimization is performed with a traditional annular illumination. SMPO works by alternating between optimizing the mask given the source, and updating the source by keeping the mask fixed, and repeating these two steps until a stopping criterion is satisfied. 


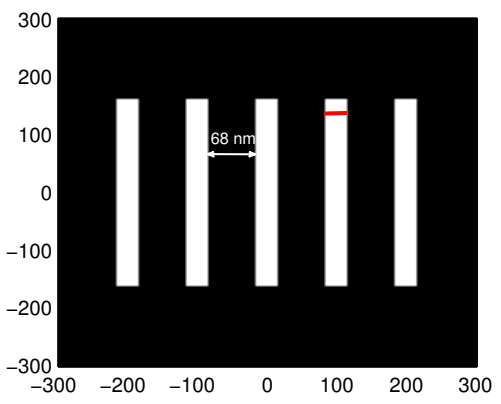

(a)

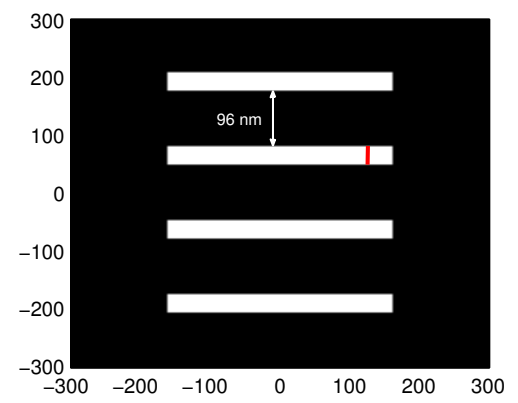

(b)

Figure 1: Two test patterns used in simulations: (a) vertical lines/spaces design and (b) horizontal lines/spaces design. Red lines mark the critical locations for measuring the process window.

\section{SIMULATION RESULTS}

To evaluate the performance of the proposed SMPO algorithm, we compare and analyze the simulation results in terms of pattern error and the process window. Two target patterns are used, namely, a vertical lines/spaces design and a horizontal lines/spaces design with different pitches, as shown in Fig. 1(a) and 1(b), respectively. Both are represented by a $151 \times 151$ matrix with a pixel size of $4 \mathrm{~nm} \times 4 \mathrm{~nm}$ and critical dimension $(\mathrm{CD})$ of $32 \mathrm{~nm}$, and the absorbers are composed of two layers, $55 \mathrm{~nm}$ of $\mathrm{Cr}(n=1.477 / k=1.762)$ below $18 \mathrm{~nm}$ of $\mathrm{CrO}$ $(n=1.965 / k=1.201)$. An annular illumination composed of $21 \times 21$ pixels with its inner annulus $\sigma_{\text {in }}=0.7$ and outer annulus $\sigma_{\text {out }}=0.9$ is adopted as the initial value for our source optimization. The parameters of the projection system are set to be $\lambda=193 \mathrm{~nm}$ and $\mathrm{NA}=1.35$.

As described previously, we firstly perform the exhaustive search at these two different pitches and find optimal $z_{9}=-0.02$ and $z_{16}=0.06$ are advantageous to both patterns. This corresponds to that mask topography induces a positive value of primary spherical aberration and a negative value of secondary spherical aberration. As a consequence, our proposed source and mask optimization is achieved under the condition of aberrated pupil with $z_{9}=0.02$ and $z_{16}=-0.06$, and current pupil wavefront distribution is shown as Fig. 2

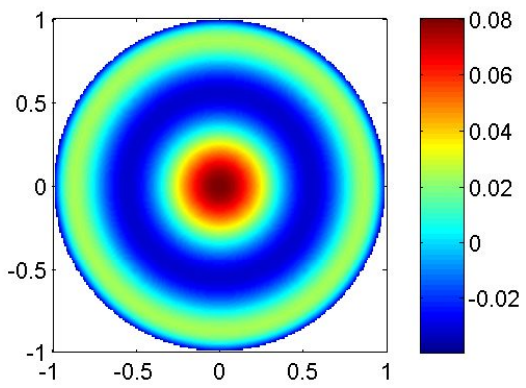

Figure 2: Current pupil wavefront distribution: a combination of primary and secondary spherical aberration.

In order to evaluate the image fidelity, we compare the optimization results using our proposed SMPO framework with an SMO with ideal pupil, where the lines/spaces pattern in Fig. 1(a) is used as input. All simulations and process window analysis in this Section are performed using the Fraunhofer IISB development and research lithography simulator Dr.LiTHO, which computes the mask near fields through the Waveguide method. ${ }^{29}$ Figures $3(\mathrm{a})-3(\mathrm{c})$ respectively display the resulting source, the optimized mask and the printed image at nominal conditions using the conventional SMO. The corresponding results from the robust SMPO with $z_{9}=0.02$ and $z_{16}=-0.06$ are given in the following row with the same structure. It is observed that printed image quality in Fig. 3(f) using the proposed SMPO including Zernike terms $z_{9}$ and $z_{16}$ is improved, 
and the pattern errors is reduced by $9 \%$ than that in Fig. 3(c). All the optimized sources are normalized by the maximum pixel intensity for better visualization.

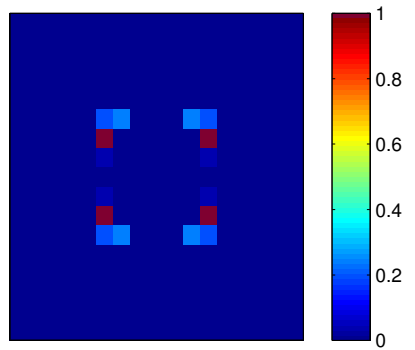

(a) Optimized source from SMO

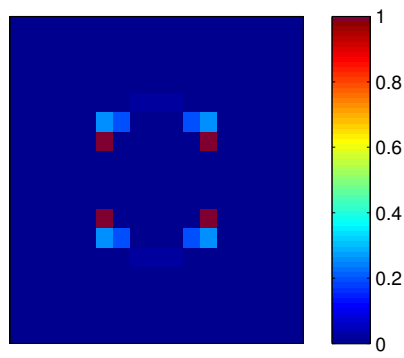

(d) Optimized source from SMPO

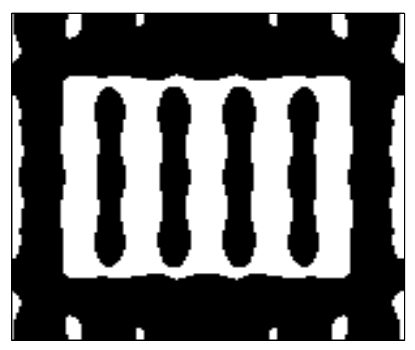

(b) Optimized mask from SMO

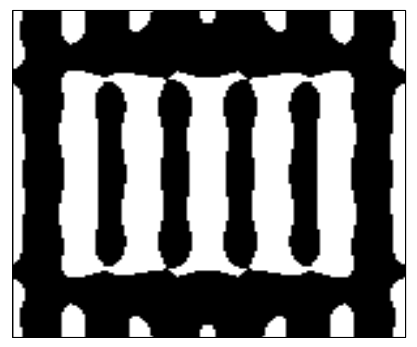

(e) Optimized mask from SMPO

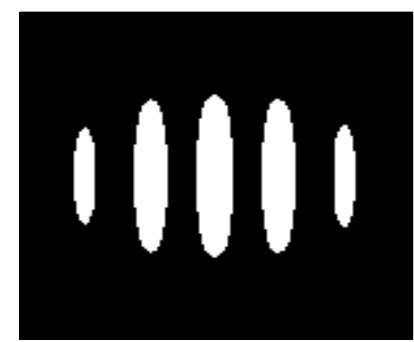

(c) Simulated output at nominal condition Pattern error $=1708$

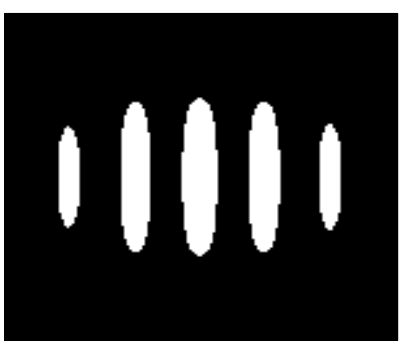

(f) Simulated output at nominal condition Pattern error $=1557$

Figure 3: Simulation results of the first test pattern.

As further evidence that the circuit pattern quality is indeed improved by SMPO, another simulation is conducted with the horizontal lines/spaces patterns in Fig. 1(b) as input, using our proposed SMPO framework and conventional SMO without applied wavefront aberrations. Figure 4 presents the corresponding results in a way similar to the above. Comparing the circuit images printed at nominal condition shown in Fig. 4(c) and 4(f), it is worth noting that not only the end regions but also the middle lines in the former have more distortions, which signifies that our method has a better resolution enhancement capacity over such regions. Especially for the two lines located in the top and bottom of the image, SMO nearly fails to print them. In contrast, they can achieve a better shape during SMPO. It can also be seen from the comparison of Figs. 4(a) and 4(d) that inclusion of Zernike term of spherical aberration in the optimization procedure can provide a more reasonable source shape, since source points along vertical axis are helpful in printing horizontal lines and spaces. ${ }^{30}$

Table 1 summarizes the measurements of the pattern errors for the two test patterns, where we compute the mask near fields with the thin mask model-Kirchhoff, and thick mask model respectively. For each model, we compare the results from two methods. To compare the performance of the methods, in a way that is as independent as possible from the different stopping criteria, we run these methods until they reach similar pattern error values in Kirchhoff model, where a fewer pattern error in rigorous model indicates an improved image fidelity. For both test patterns, when both two methods produce similar pattern errors, SMPO can also achieve a better performance in rigorous simulation, reducing about $9 \%$ and $32 \%$ pattern errors, respectively. In other words, incorporating sensitive aberrations terms in SMPO gives a more robust design against the mask-induced aberrations.

Since there is another kind of joint source, mask and pupil optimization algorithm, which manipulates the pupil after source and mask optimization to compensates the mask induced effects. We run the simulations using 


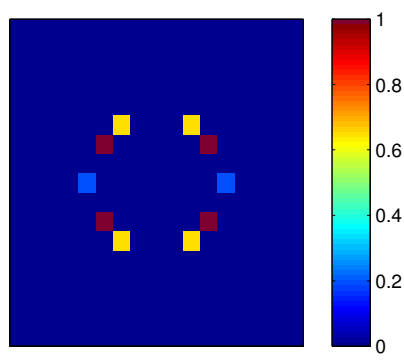

(a) Optimized source from SMO
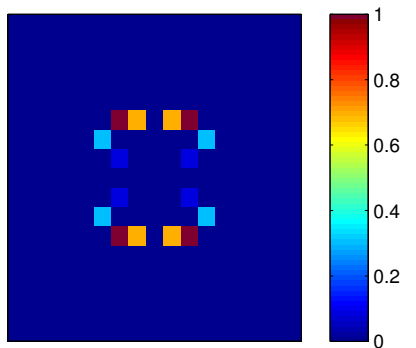

(d) Optimized source from SMPO

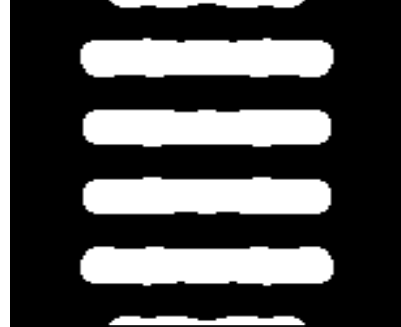

(b) Optimized mask from SMO

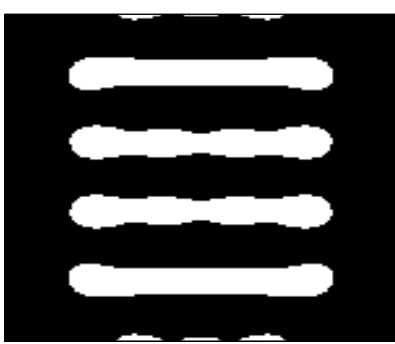

(e) Optimized mask from SMPO

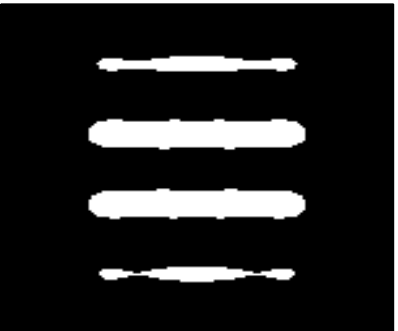

(c) Simulated output at nominal condition Pattern error $=1280$

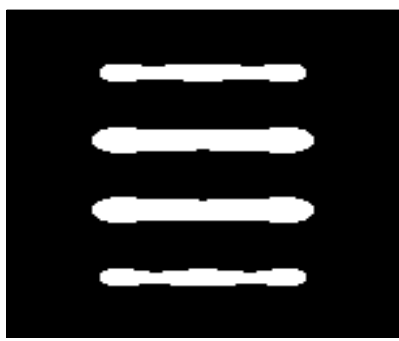

(f) Simulated output at nominal condition Pattern error $=866$

Figure 4: Simulation results of the second test pattern.

Table 1: Comparison of performance with different models and methods.

\begin{tabular}{|c|c|c|c|c|}
\hline Test patterns & Pitch & Model & Methods & PE (pixels) \\
\hline \multirow{3}{*}{ Vertical lines } & \multirow{3}{*}{$100 \mathrm{~nm}$} & \multirow{2}{*}{ Kirchhoff model } & SMO & 80 \\
& & SMPO & 76 \\
\cline { 3 - 5 } & & \multirow{2}{*}{ Rigorous model } & SMO & 1708 \\
& & SMPO & 1557 \\
\hline \multirow{3}{*}{ Horizontal lines } & \multirow{2}{*}{$128 \mathrm{~nm}$} & \multirow{2}{*}{ Kirchhoff model } & SMO & 148 \\
\cline { 3 - 5 } & & \multirow{2}{*}{ Rigorous model } & SMO & 182 \\
\cline { 3 - 5 } & & & SMPO & 866 \\
\hline
\end{tabular}

the optimal sources and masks resulted from SMO to see the pattern error change versus $z_{9}$ and $z_{16}$ coefficients, as plotted in Fig. 5. For both two cases, it can be observed from Figs. 5 (a) and (b) that when $z_{9}$ and $z_{16}$ changes within $[-0.1,0.1]$, all the pattern errors resulted from SMO approach are more than those from SMPO method. In other words, even if pupil wavefront receives the further control after SMO, the proposed SMPO algorithm incorporating pupil spherical aberrations into source and mask optimization process still can deliver a better design. This result is related to the fact that a combination of $z_{9}$ and $z_{16}$ in the way in our method would provide compensation effectively. Besides, the pattern error varies in a larger range with the change of $z_{9}$ value than that versus $z_{16}$ variation, as illustrated by the red and the magenta curves in Fig. 5. This agrees with previous study that spherical aberrations have the highest sensitivity with primary spherical aberration the most sensitive. ${ }^{26}$ 


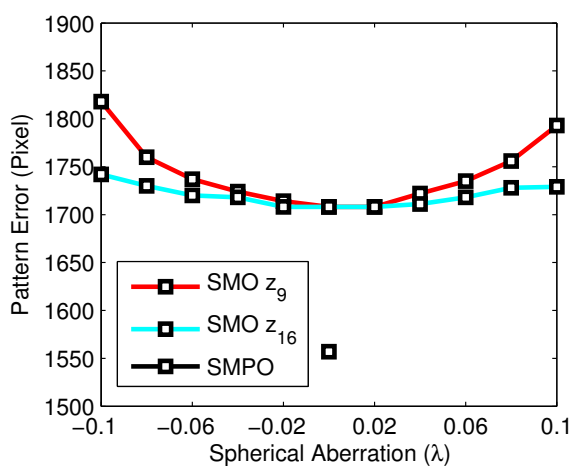

(a)

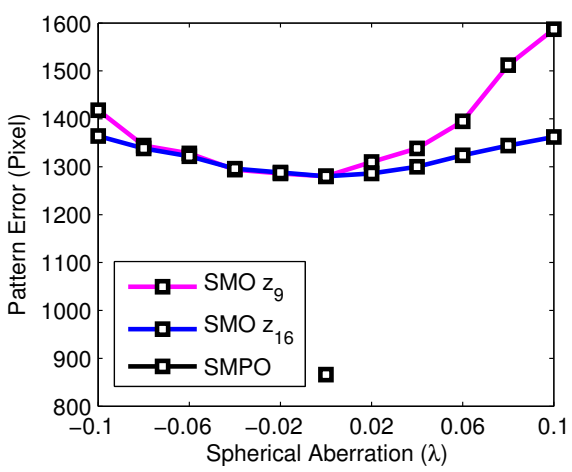

(b)

Figure 5: Simulated pattern errors at different spherical aberration values $\left(z_{9} / z_{16}\right)$ for (a) vertical lines at $100 \mathrm{~nm}$ pitch, and (b) horizontal lines at $128 \mathrm{~nm}$ pitch.

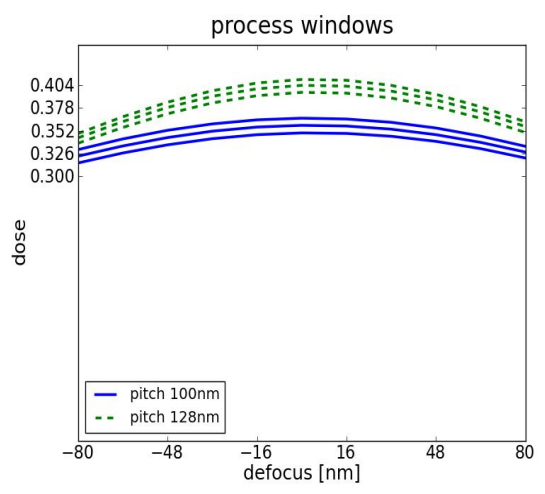

(a)

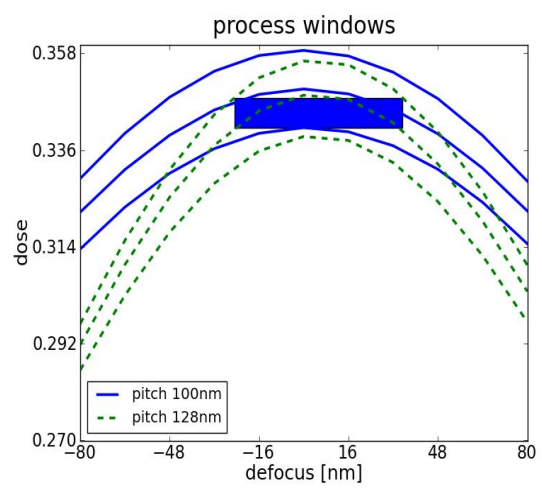

(b)

Figure 6: Simulated process window of $32 \mathrm{~nm}$ vertical lines at $100 \mathrm{~nm}$ pitch and $32 \mathrm{~nm}$ horizontal lines at $128 \mathrm{~nm}$ pitch with (a) conventional SMO without pupil aberration and (b) SMPO.

After evaluating the image quality of different algorithms, we can now assess the robustness of the proposed algorithm in terms of the process window. Fig. 6 depicts the average exposure-defocus window comparison, and the three lines for one pitch represent the dose values to create features with the target linewidth and with a deviation of $\pm 10 \%$ from the target linewidth, respectively. The minimum feature size (also the width of the feature) is chosen as the critical regions, as marked by the red lines in Fig. 1 (a) and (b). The usable depth of focus is evaluated by checking the largest acceptable defocus range of an rectangular tangent with the color curves at a particular dose. If we fix the exposure latitude (EL) condition at 2\%, from Fig. 6(a), $100 \mathrm{~nm}$ pitch vertical lines have no uDOF with $128 \mathrm{~nm}$ horizontal lines. In comparison, a larger uDOF is able to be observed in the Fig. 6(b), where the proposed SMPO including both $z_{9}$ and $z_{16}$ coefficients increases the process capability by producing a $60 \mathrm{~nm}$ larger defocus range than conventional SMO method, demonstrating enhanced process robustness. Such results are reasonable because the consideration of pupil wavefront in the form of spherical aberration in the optimization procedure can compensate for mask topography degraded uDOF, and another possible reason is that the optimal source in Fig. 4(d) with more intensity on the vertical-oriented poles can stretch the lines closer to the original design (Fig. 1(b)). 


\section{CONCLUSIONS}

In conclusion, a mutual SMPO algorithm is presented for robust source and mask design in optical lithography. We investigate how to incorporate primary and secondary spherical aberrations terms in the optimization procedure to compensate for thick mask topography effects. The application of the approach to 1D lines/spaces pattern with different pitches of vertical and horizontal configurations shows advantages such as high image fidelity and improved process robustness. This allows the research on pupil plane, together with source and mask optimization to be a prime candidate for computational lithography with the increasing severity of mask-induced effects.

\section{ACKNOWLEDGMENTS}

This work was supported in part by the UGC Areas of Excellence project Theory, Modeling, and Simulation of Emerging Electronics, and by the State Key Lab of Digital Manufacturing Equipment and Technology under Project DMETKF2013003. The authors would also like to acknowledge Fraunhofer Institute for Integrated Systems and Device Technology for the use of Dr.LiTHO.

\section{REFERENCES}

[1] Yang, S.-H., Jia, N., Shim, S., Vengertsev, D., Kim, Y., Choi, J., and Kang, H.-K., "The effect of mask and source complexity on source mask optimization," in [Optical Microlithography XXVI], Conley, W., ed., Proc. SPIE 8683, 86830C (2013).

[2] Agudelo, V., Evanschitzky, P., Erdmann, A., Fühner, T., Shao, F., Limmer, S., and Fey, D., "Accuracy and performance of 3D mask models in optical projection lithography," in [Optical Microlithography XXIV], Dusa, M. V., ed., Proc. SPIE 7973, 797300 (2011).

[3] Finders, J. and Hollink, T., "Mask 3D effects: impact on imaging and placement," in [27th European Mask and Lithography Conference], Behringer, U. F., ed., Proc. SPIE 7985, 79850I (2011).

[4] Coskun, T. H., Dai, H., Huang, H.-T., Kamat, V., and Ngai, C., "Accounting for mask topography effects in source-mask optimization for advanced nodes," in [Optical Microlithography XXIV], Dusa, M. V., ed., Proc. SPIE 7973, 79730P (2011).

[5] Lam, E. Y. and Wong, A. K., "Computation lithography: virtual reality and virtual virtuality," Opt. Express 17, 12259-12268 (2009).

[6] Lam, E. Y. and Wong, A. K., "Nebulous hotspot and algorithm variability in computation lithography," J. Micro/Nanolith. MEMS MOEMS 9, 033002 (2010).

[7] Jia, N. and Lam, E. Y., "Machine learning for inverse lithogrpahy: using stochastic gradient descent for robust photomask synthesis," J. Opt 12, 045601 (2010).

[8] Fakhry, M., Granik, Y., Adam, K., and Lai, K., "Total source mask optimization: high-capacity, resist modeling, and production-ready mask solution," in [Photomask Technology 2011], Maurer, W. and Abboud, F. E., eds., Proc. SPIE 8166, 81663M (2011).

[9] Ma, X., Han, C., Li, Y., Dong, L., and Arce, G. R., "Pixelated source and mask optimization for immersion lithography," J. Opt. Soc. Am. A 30, 112-123 (2013).

[10] Li, J., Liu, S., and Lam, E. Y., "Efficient source and mask optimization with augmented Lagrangian methods in optical lithography," Opt. Express 21, 8076-8090 (2013).

[11] Sears, M. K., Fenger, G., Mailfert, J., and Smith, B., "Extending SMO into the lens pupil domain," in [Optical Microlithography XXIV], Dusa, M. V., ed., Proc. SPIE 7973, 79731B (2011).

[12] Agudelo, V., Evanschitzky, P., Erdmann, A., and Fühner, T., "Evaluation of various compact mask and imaging models for the efficient simulation of mask topography effects in immersion lithography," in [Optical Microlithography XXV], Conley, W., ed., Proc. SPIE 8326, 832609 (2012).

[13] Chan, S. H., Wong, A. K., and Lam, E. Y., "Initialization for robust inverse synthesis of phase-shifting masks in optical projection lithography," Opt. Express 16, 14746-14760 (2008).

[14] Jia, N., Wong, A. K., and Lam, E. Y., "Robust mask design with defocus variation using inverse synthesis," in [Lithography Asia], Chen, A. C., Lin, B., and Yen, A., eds., Proc. SPIE 7140, 71401W (2008). 
[15] Shen, Y., Wong, N., and Lam, E. Y., "Level-set-based inverse lithography for photomask synthesis," Opt. Express 17, 23690-23701 (2009).

[16] Choy, S. K., Jia, N., Tong, C. S., Tang, M. L., and Lam, E. Y., "A robust computational algorithm for inverse photomask synthesis in optical projection lithography," SIAM J. Imaging Science 5, 625-651 (2012).

[17] Wong, A. K., [Optical Imaging in Projection Microlithography], SPIE, Washington (2005).

[18] Shen, Y., Wong, N., and Lam, E. Y., "Aberration-aware robust mask design with level-set-based inverse lithography," in [Photomask and Next-Generation Lithography Mask Technology XVII], Hosono, K., ed., Proc. SPIE 7748, $77481 \mathrm{U}$ (2010).

[19] Shen, Y., Jia, N., Wong, N., and Lam, E. Y., "Robust level-set-based inverse lithography," Opt. Express 19, $5511-5521$ (2011).

[20] Jia, N. and Lam, E. Y., "Pixelated source mask optimization for process robustness in optical lithography," Opt. Express 19, 19384-19398 (2011).

[21] Li, J., Shen, Y., and Lam, E. Y., "Hotspot-aware fast source and mask optimization," Opt. Express 20, 21792-21804 (2012).

[22] Poonawala, A. and Milanfar, P., "Mask design for optical microlithography - an inverse imaging problem," IEEE Trans. Image Process. 16, 774-788 (2007).

[23] Dirksen, P., Braat, J. J., Janssen, A. J., and Leeuwestein, A., "Aberration retrieval for high-NA optical systems using the extended Nijboer-Zernike theory," in [Optical Microlithography XVIII], Smith, B. W., ed., Proc. SPIE 5754, 262-273 (2005).

[24] Mack, C., [Fundamental Principles of Optical Lithography: The Science of Microfabrication], WILEY, West Sussex (2007).

[25] Evanschitzky, P., Shao, F., Fühner, T., and Erdmann, A., "Compensation of mask induced aberrations by projector wavefront control," in [Optical Microlithography XXIV], Dusa, M. V., ed., Proc. SPIE 7973, 797329 (2011).

[26] Sears, M. K., Bekaert, J., and Smith, B. W., "Lens wavefront compensation for 3D photomask effects in subwavelength optical lithography," Appl. Opt. 52, 314-322 (2013).

[27] Jia, N., Wong, A. K., and Lam, E. Y., "Regularization of inverse photomask synthesis to enhance manufacturability," in [Lithography Asian], Chen, A. C., Han, W.-S., Lin, B. J., and Yen, A., eds., Proc. SPIE 7520, 75200E (2009).

[28] Nocedal, J. and Wright, S. J., [Numerical Optimization], Springer, New York (2006 (second edition)).

[29] Erdmann, A., Shao, F., Evanschitzky, P., and Fühner, T., "Mask-topography-induced phase effects and wave aberrations in optical and extreme ultraviolet lithography," J. Vac. Sci. Technol. B 28, C6J1-C6J7 (2010).

[30] Deng, Y., Zou, Y., Yoshimoto, K., Ma, Y., Tabery, C. E., Kye, J., Capodieci, L., and Levinson, H. J., "Considerations in source-mask optimization for logic applications," in [Optical Microlithography XXIII], Dusa, M. V. and Conley, W., eds., Proc. SPIE 7640, 76401J (2010). 